\title{
Exosome-derived galectin-9 may be a novel predictor of rejection and prognosis after liver transplantation*
}

\author{
Ai-bin ZHANG ${ }^{\S 1}$, Yi-fan PENG ${ }^{\S 1,2}$, Jun-jun JIA ${ }^{1}$, Yu NIE ${ }^{3}$, Shi-yu ZHANG ${ }^{1,2}$, \\ Hai-yang XIE ${ }^{1,2}$, Lin ZHOU ${ }^{1,2}$, Shu-sen ZHENG ${ }^{\dagger 1,2}$ \\ ${ }^{I}$ Division of Hepatobiliary and Pancreatic Surgery, Department of Surgery, the First Affiliated Hospital, \\ School of Medicine, Zhejiang University, Hangzhou 310003, China \\ ${ }^{2}$ NHFPC Key Laboratory of Combined Multi-organ Transplantation, the First Affiliated Hospital, Zhejiang University, Hangzhou 310003, China \\ ${ }^{3}$ Organ Transplant Center, the First Affiliated Hospital, Sun Yat-sen University, Guangzhou 510080, China \\ †E-mail: shusenzheng@zju.edu.cn \\ Received Jan. 29, 2019; Revision accepted Apr. 14, 2019; Crosschecked May 23, 2019
}

\begin{abstract}
Acute cellular rejection (ACR) remains a major concern after liver transplantation. Predicting and monitoring acute rejection by non-invasive methods are very important for guiding the use of immunosuppressive drugs. Many studies have shown that exosomes and their contents are potential biomarkers for various liver diseases. Here, we identify and validate the role of exosomes and galectin-9 in ACR after liver transplantation. Exosomes were isolated from three sets of paired patients, with and without $A C R$, and the proteins within the exosomes were isolated and identified. Candidate proteins were then validated using a tissue microarray containing resected liver samples from 73 ACR and 63 non-rejection patients. Finally, protein expression and clinical manifestations were included in KaplanMeier survival and Cox regression analyses. Circulating exosomes were isolated from ACR and non-rejection patients and characterized using transmission electron microscopy and western blotting for CD63/CD81. Western blotting experiments revealed higher levels of galectin- 9 protein in circulating exosomes from ACR recipients. Immunohistochemical analysis of the tissue microarray showed that the expression of galectin- 9 in resected liver was significantly higher in the ACR group than in the non-rejection group $(P<0.05)$. Higher levels of galectin-9 expression in resected livers were associated with poorer prognosis $(P<0.05)$. Exosome-derived galectin-9 may be a novel predictor of rejection and prognosis after liver transplantation.
\end{abstract}

Key words: Liver transplantation; Acute cellular rejection; Exosome; Galectin-9 https://doi.org/10.1631/jzus.B1900051

CLC number: R446

\footnotetext{
${ }^{\ddagger}$ Corresponding author

$\S$ The two authors contributed equally to this work

* Project supported by the China Postdoctoral Science Foundation (No. 2017M610374), the Zhejiang Health Technology Project (No. 2019RC153), the Zhejiang Provincial Natural Science Foundation of China (No. Y17H160118), and the National Natural Science Foundation of China (No. 91542205)

(b) ORCID: Ai-bin ZHANG, https://orcid.org/0000-0002-1149-4347 (C) Zhejiang University and Springer-Verlag GmbH Germany, part of Springer Nature 2019
}

\section{Introduction}

Although liver transplantation is a life-saving treatment for end-stage liver disease, acute cellular rejection (ACR) remains an important complication after liver transplantation (Zhou et al., 2015; Vitalone et al., 2016). ACR leads to increased use of immunosuppressive drugs, which may contribute to higher rates of infection and cancer recurrence in recipients. Avoiding unnecessary immunosuppression requires biomarkers that can predict and identify the risk of 
ACR (Pike et al., 2016). There is currently no effective way to detect rejection besides direct diagnosis with allograft biopsies. Predicting and monitoring acute rejection by non-invasive methods are very important for guiding the use of immunosuppressive drugs for transplant recipients.

Many studies have focused on the identification of non-invasive biomarkers for ACR. Biomarkers that have been identified thus far for predicting and monitoring ACR include: cell-based assays, such as allospecific $\mathrm{CD} 154^{+}$cytotoxic memory $\mathrm{T}$ cells (Ashokkumar et al., 2017); the ratio of circulating $\mathrm{CD}^{+} \mathrm{CD} 25^{+} \mathrm{FoxP}^{+}{ }^{+}$regulatory $\mathrm{T}$ cells/Th17 cells (Wang et al., 2014) and protein death 1-expressing $\mathrm{T}$ cells (Pike et al., 2016); immune function markers, such as CD38, CD69, CD95, and CD154 on peripheral $\mathrm{T}$ lymphocytes (Boix et al., 2016); and the levels of cytokines and chemokines, such as interleukin 10 (IL-10), IL-17, CXCL10 (Kim et al., 2016), CXCL9 (Raschzok et al., 2015), and interferon $\gamma$ (IFN- $\gamma$ ) (Sood et al., 2017; Meszaros et al., 2019). However, these biomarkers are usually insensitive and nonspecific and do not reflect the severity of ACR or support early diagnosis.

Exosomes are vesicles of endosomal origin (20$120 \mathrm{~nm}$ in diameter), characterized by surface markers including CD63 and CD81. They have been shown to play an important role in intercellular communication. They can transfer their biochemical contents (proteins, microRNAs (miRNAs), messenger RNAs (mRNAs), DNA, and lipids) directly to target cells in their vicinity or at a distance, through endocytosis or membrane receptors. Exosomes have been extensively studied for the diagnosis and treatment of many diseases, including cancer and ischemia, and for the promotion of tissue regeneration (Kerr et al., 2018; Lazar et al., 2018; Mousavi et al., 2019). Some researchers have studied the relationship between ACR and exosomes. Vallabhajosyula et al. (2017) focused on exosome biomarkers in islet transplantation, showing that donor-specific exosomes can be detected in the circulation of recipients, and these exosomes, with distinct protein and RNA profiles, are present prior to hyperglycemia and can be used to detect ACR. However, few studies have investigated the use of exosomes and their contents to predict and monitor ACR after liver transplantation.
Galectin-9 is a $36-\mathrm{kDa}$ lectin protein, which is involved in the progression of many diseases and may act as an important biomarker for diagnosis and treatment (Saitoh et al., 2012; Kurose et al., 2013; van den Hoogen et al., 2018). Kidney transplantation patients with rejection show significantly higher levels of galectin-9 than those without rejection (Naka et al., 2009; Li et al., 2018). Qiao et al. (2014) reported an increase galectin-9 expression during acute rejection in a rat model of lung transplantation. Galectin-9 is a ligand for transmembrane immunoglobulin mucin domain 3 (Tim-3). Patients with steroid-resistant rejection have higher urinary Tim-3 levels than steroidsensitive patients (Chen et al., 2017). These results suggest that galectin-9 may have an important role in ACR. In this study, we aimed to assess the value of exosomes and their derived proteins, such as galectin-9, in predicting and monitoring ACR.

\section{Methods and materials}

\subsection{Patients and samples}

Seventy-three patients with ACR after liver transplantation in the First Affiliated Hospital of Zhejiang University (Hangzhou, China), from 2006 to 2014, were enrolled in this study. Sixty-three patients without rejection after liver transplantation during the same period were chosen as controls. All patients were diagnosed with end-stage liver disease caused by benign disease. Resected liver tissue samples were obtained and embedded in paraffin for further study. All recipients received consecutive tacrolimus-based immunosuppressive regimens (corticosteroids and mycophenolate mofetil). Tacrolimus was initiated at $2-3 \mathrm{mg} / \mathrm{d}$ for the first month after transplantation and was then titrated down to $5-10 \mathrm{ng} / \mathrm{mL}$ over the next few months. Methylprednisolone was administered at $1000 \mathrm{mg}$ in the perioperative period and was slowly withdrawn in the first month after transplantation. Mycophenolate mofetil was administered at $500 \mathrm{mg}$, twice a day after transplantation. All ACR diagnoses were made by clinical indications of elevated liver enzyme levels and pathological examination of acute rejection using the Banff criteria (Demetris et al., 2016). All experiments were approved by the Ethics Review Committee of the First Affiliated Hospital, Zhejiang University (approval number: ref 2017-336). 


\subsection{Exosome isolation and identification}

Total exosomes were isolated from serum using a total exosome isolation kit (Life Technologies, Carlsbad, CA, USA) according to the manufacturer's instructions. In brief, an initial centrifugation was performed at $2000 \mathrm{~g}$ (room temperature) for $30 \mathrm{~min}$ for each sample and the supernatant was collected. Total exosome isolation reagents were then added at $1 / 5$ of the starting sample volume. Mixtures were vortexed, incubated at $4{ }^{\circ} \mathrm{C}$ for up to $30 \mathrm{~min}$, and then centrifuged at $10000 \mathrm{~g}$ for $10 \mathrm{~min}$ at room temperature to precipitate exosome pellets. The exosome fraction was resuspended using $1 / 2 \times$ phosphate buffered saline (PBS) and stored at $-80^{\circ} \mathrm{C}$ until further analysis. The morphological characteristics and particle size of the exosomes were determined by transmission electron microscopy (TEM). The exosome marker molecules, CD63 and CD81, were detected by western blotting analysis.

\subsection{Transmission electron microscopy}

The morphological characteristics and particle size of exosomes were examined using standard TEM procedures. Briefly, exosome suspensions were fixed in $2.5 \%(\mathrm{v} / \mathrm{v})$ glutaraldehyde $\left(4^{\circ} \mathrm{C}, \mathrm{pH} 7.4\right)$, postfixed in $1 \%(\mathrm{v} / \mathrm{v})$ osmium tetroxide, applied onto copper mesh grids, and stained with $1 \%(\mathrm{v} / \mathrm{v})$ phosphotungstanic acid. The grids were dried at room temperature for $10 \mathrm{~min}$ and viewed at $10000 \times$ magnification using an electron microscope (Philips, Eindhoven, the Netherlands).

\subsection{Western blotting analysis}

Western blotting was performed according to the manufacturer's instructions. In brief, total protein was extracted from exosomes. Proteins were separated by sodium dodecyl sulfate-polyacrylamide gel electrophoresis (SDS-PAGE) and transferred to polyvinylidene difluoride membranes. After blocking, the blots were incubated with an anti-galectin-9 antibody (Abcam, Cambridge, UK) overnight at $4{ }^{\circ} \mathrm{C}$. Membranes were then incubated with secondary antibodies. After washing the membranes, proteins were visualized using an enhanced chemiluminescence (ECL) detection system. Anti-CD81 and -CD63 antibodies (Abcam) were used as internal controls.

\subsection{Immunohistochemical analysis of liver tissues}

Resected liver specimens from all transplant recipients enrolled in the study were embedded in paraffin wax and used by Outdo Biotech Co., Ltd. (Shanghai, China) to prepare tissue microarrays. A primary antibody against galectin-9 was used for immunohistochemical analysis. All samples were randomly placed on microarrays and analyzed by two independent pathologists. Immunohistochemical staining of galectin-9 was performed on the microarray sections. In brief, tissue microarray slides were deparaffinized, rehydrated, and then treated for antigen retrieval. Endogenous peroxidase activity was then blocked. After washing and incubating overnight at $4{ }^{\circ} \mathrm{C}$ with a primary antibody against galectin-9 (1:600 dilution, Abcam), the slides were incubated with secondary antibody for $30 \mathrm{~min}$. They were then immersed in diaminobenzidine and hematoxylin. Data were analyzed using a ScanScope XT slide scanner (Aperio, CA, USA). Each sample on the tissue microarray was semi-quantitatively scored for staining intensity and the percentage of immunoreactive cells by two specialists blinded to patient information. Staining intensity was scored as " 0 " for no staining, "1" for weak staining, " 2 " for moderate staining, and " 3 " for strong staining. The percentage of immunoreactive cells was scored as " 0 " for $<20 \%$ positivity, " 1 " for $21 \%-40 \%$, " 2 " for $41 \%-60 \%$, " 3 " for $61 \%-80 \%$, and " 4 " for $81 \%-100 \%$. The total score for galectin-9 immunostaining was calculated by multiplying the percentage of immunoreactive cells score by the staining intensity score. Total scores ranged from 0 to 12 . We defined a galectin- 9 expression score $\geq 8$ as high expression.

\subsection{Statistical analysis}

All data are presented as the number or mean \pm standard deviation (SD). A Student's $t$ test or $\chi^{2}$ test was used to analyze differences between two groups. Kaplan-Meier curves were used to study patient survival, along with the log-rank test. The multivariate analysis was based on Cox regression analyses. All statistical analyses were performed using SPSS software (Version 23.0; SPSS Inc., Chicago, IL, USA). A two-tailed $P$-value of $<0.05$ was considered statistically significant. 


\section{Results}

\subsection{Galectin-9 expression in serum exosomes of patients with ACR}

Peripheral blood samples were taken from three sets of paired patients, with and without ACR. The clinical characteristics of the rejection and nonrejection patients are shown in Table 1. Exosomes were isolated and analyzed by electron microscopy. They exhibited a typical morphology, with a mean vesicle diameter of 20-120 nm (Fig. 1). The amount of galectin-9 in exosomes was quantified. Compared with the non-rejection group, the rejection group showed significantly higher levels of galectin-9 in exosomes $(P<0.05$; Fig. 2$)$. These results showed that galectin-9 levels in exosomes are associated with ACR after liver transplantation.

\subsection{Galectin-9 expression in the livers of patients with ACR}

Since we demonstrated an association between liver rejection and galectin-9 expression in exosomes, we hypothesized that the intrinsic galectin- 9 levels of transplant recipients are associated with post-transplant rejection. Resected livers from transplant recipients provide non-invasive materials for determining the

Table 1 Clinical characteristics of rejection and nonrejection patients used for exosome and galectin-9 expression analyses

\begin{tabular}{ccccc}
\hline Patient & $\begin{array}{c}\text { Age } \\
\text { (years) }\end{array}$ & Gender & Diagnosis & $\begin{array}{c}\text { Time until } \\
\text { rejection (d) }\end{array}$ \\
\hline Rejection & & & & \\
Patient 1 & 55 & F & Cirrhosis & 9 \\
Patient 2 & 44 & F & Cirrhosis & 12 \\
Patient 3 & 34 & M & Cirrhosis & 11 \\
Non-rejection & & & & \\
Patient 1 & 61 & F & Cirrhosis & \\
Patient 2 & 47 & F & Cirrhosis & \\
Patient 3 & 37 & M & Cirrhosis & \\
\hline
\end{tabular}

F: female; M: male



Fig. 1 Identification of exosome with electron microscopy The vesicles (arrows) exhibit typical exosome morphology, with a mean vesicle diameter of $20-120 \mathrm{~nm}$ association between intrinsic biomarker profiles of patients and their primary disease. To test this hypothesis, we enrolled 136 patients in the study. Seventy-three patients had ACR after liver transplantation (rejection group) and 63 patients did not (non-rejection group). The demographics and clinical characteristics of these patients are summarized in Table 2. Importantly, the rejection and non-rejection groups did not differ significantly in respect to gender, model for end-stage liver disease (MELD) scores, serum creatinine concentration, or ABO compatibility. However, patients in the rejection group were significantly younger and had higher Child-Pugh scores than patients in the non-rejection group (both $P<0.05$; Table 2). Immunohistochemical analysis of a tissue microarray was performed to evaluate the levels of galectin-9 expression in the resected liver tissues from the two groups. Compared with the nonrejection group, galectin-9 expression was significantly higher in the rejection group $(P<0.05$; Fig. 3$)$.

Table 2 Demographics and clinical characteristics of patients for galectin-9 expression analysis in validation study

\begin{tabular}{llcl}
\hline \multicolumn{1}{c}{ Characteristics } & $\begin{array}{c}\text { Rejection } \\
(n=73)\end{array}$ & $\begin{array}{c}\text { Non-rejection } \\
(n=63)\end{array}$ & $P$-value \\
\hline Age (years) & $40.4 \pm 9.6$ & $46.6 \pm 10.3$ & $<0.05$ \\
Gender & & & \\
$\quad$ Male & $59(80.8 \%)$ & $56(88.9 \%)$ & NS \\
$\quad$ Female & $14(19.2 \%)$ & $7(11.1 \%)$ & \\
MELD score & $23.8 \pm 10.0$ & $21.8 \pm 10.4$ & $\mathrm{NS}$ \\
Child-Pugh score & $10.2 \pm 2.3$ & $9.3 \pm 2.3$ & $<0.05$ \\
Serum creatinine & $83.3 \pm 64.3$ & $108.9 \pm 140.2$ & $\mathrm{NS}$ \\
$\quad(\mu \mathrm{mol} / \mathrm{L})$ & & & \\
ABO compatibility (\%) & $62(84.9 \%)$ & $51(81.0 \%)$ & $\mathrm{NS}$ \\
Time until rejection (d) & $11.2 \pm 7.5$ & & \\
\hline $\begin{array}{l}\text { Data are expressed as number (percentage) or mean } \pm \text { SD. NS: not } \\
\text { significant; MELD: model for end-stage liver disease }\end{array}$ &
\end{tabular}

(a)

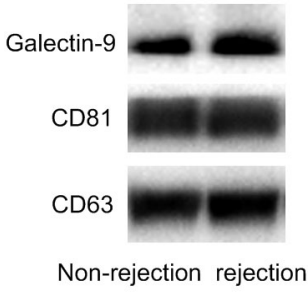

(b)

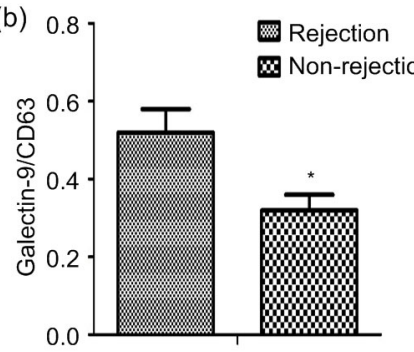

Fig. 2 Galectin-9 expression in serum exosomes (a) Western blotting assay of galectin- 9 expression in exosomes from non-rejection and rejection patients. (b) Comparison of band intensity in western blots. The data are expressed as mean $\pm \operatorname{SD}(n=3) .{ }^{*} P<0.05$, vs. rejection group 


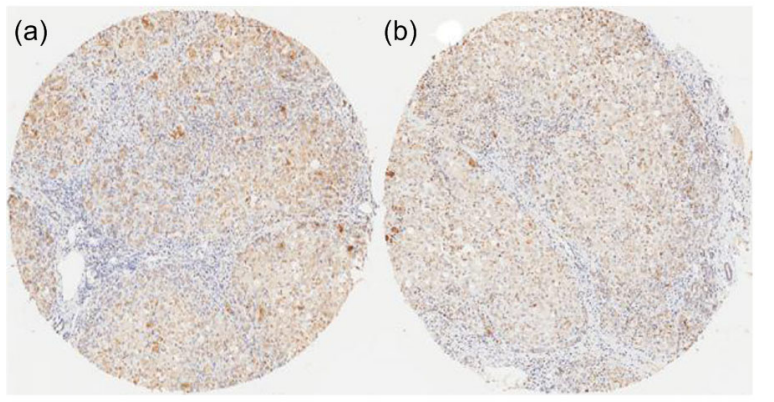

(c)

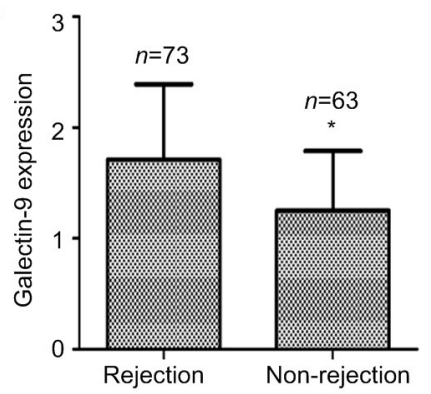

Fig. 3 Galectin-9 expression in resected livers

(a) Typical immunohistochemistry result from the rejection group. (b) Typical immunohistochemistry result from the non-rejection group. (c) Comparison of galectin-9 expression scores in the two groups. The data are expressed as mean \pm SD. ${ }^{*} P<0.05$, vs. rejection group

\subsection{Prediction of recipient survival by galectin-9 expression}

According to the level of galectin-9 expression in resected livers, patients were divided into a high expression group (expression score $\geq 8$ ) and a low expression group (expression score $<8$ ), based on the total immunostaining score. Twenty-eight cases were classified as high expression and 108 cases were classified as low expression. The two groups did not differ significantly in age, gender, MELD score, or Child-Pugh score (Table 3). Survival analysis showed that the low expression group had significantly higher survival rate than the high expression group $(P<0.05$; Fig. 4).

\subsection{Association of galectin-9 with long-term survival}

Various factors were associated with recipient survival after transplantation. In this study, seven factors were identified for multivariate analysis, including age, gender, serum creatinine concentration, ABO compatibility, MELD score, Child-Pugh status, and galectin-9 expression score. After multivariate regression analysis, galectin-9 expression score in resected livers was significantly associated with patient survival $(P<0.05$; Table 4$)$.

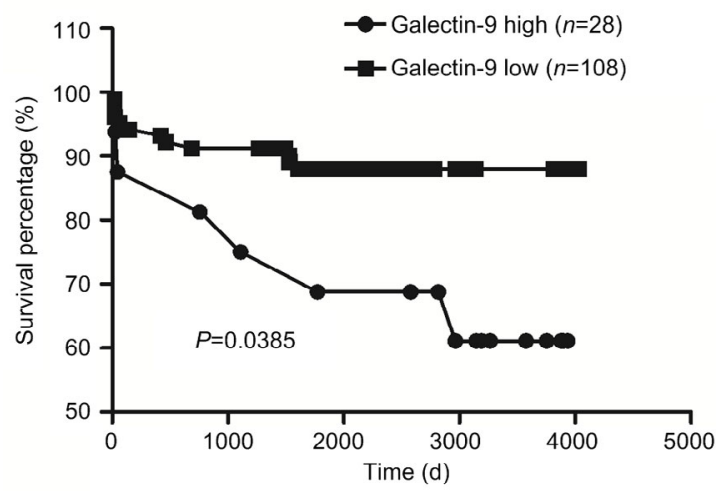

Fig. 4 Survival of the high and low galectin-9 expression groups

Galectin-9 expression in diseased liver predicts transplant recipient, based on total immunostaining score

Table 3 Characteristics of transplant recipients in the high and low galectin-9 expression groups

\begin{tabular}{|c|c|c|c|c|}
\hline Group & $\begin{array}{c}\text { Age } \\
\text { (years) }\end{array}$ & $\begin{array}{c}\text { Gender } \\
(\mathrm{M} / \mathrm{F})\end{array}$ & $\begin{array}{l}\text { Child-Pugh } \\
\text { score }\end{array}$ & $\begin{array}{c}\text { MELD } \\
\text { score }\end{array}$ \\
\hline High $(n=28)$ & $42.4 \pm 9.2$ & $22 / 6$ & $10.3 \pm 2.1$ & $24.4 \pm 10.6$ \\
\hline Low $(n=108)$ & $43.5 \pm 10.6$ & $93 / 15$ & $9.7 \pm 2.4$ & $22.5 \pm 10.2$ \\
\hline$P$-value & NS & $\mathrm{NS}$ & $\mathrm{NS}$ & NS \\
\hline
\end{tabular}

F: female; M: male; NS: not significant

Table 4 Multivariate analysis of overall survival in all transplant recipients

\begin{tabular}{lrrrrcc}
\hline \multicolumn{1}{c}{ Variable } & \multicolumn{1}{c}{$B$} & \multicolumn{1}{c}{ SE } & \multicolumn{1}{c}{ Wald } & \multicolumn{1}{c}{$d f$} & Sig. & $\operatorname{Exp}(B)$ \\
\hline Age $(>50$ years vs. $\leq 50$ years) & -0.685 & 0.537 & 1.627 & 1 & 0.202 & 0.504 \\
Gender (female vs. male) & -2.044 & 0.537 & 14.488 & 1 & 0.000 & 0.130 \\
Serum creatinine $(>130 \mu \mathrm{mol} / \mathrm{L}$ vs. $\leq 130 \mu \mathrm{mol} / \mathrm{L})$ & 13.447 & 846.409 & 0.000 & 1 & 0.987 & 691446 \\
ABO compatibility & -1.039 & 1.054 & 0.972 & 1 & 0.324 & 0.354 \\
MELD score $(\leq 22$ vs. $>22)$ & 0.714 & 0.604 & 1.399 & 1 & 0.237 & 2.043 \\
Child-Pugh status (Child C vs. Child A and Child B) & -0.811 & 0.680 & 1.423 & 1 & 0.233 & 0.444 \\
Galectin-9 expression (high vs. low) & -0.666 & 0.572 & 1.355 & 1 & 0.044 & 0.514 \\
\hline
\end{tabular}

$B$ : coefficient; SE: standard error; Wald: Wald statistic; $d f$ : degrees of freedom; Sig.: significance level; $\operatorname{Exp}(B)$ : $\operatorname{exponenti-}$ ation of the $B$ 


\section{Discussion}

In the present study, high levels of galectin-9 expression were found in exosomes and resected livers of patients with ACR, and galectin-9 expression was found to be an independent factor for recipient survival. These results indicate that exosome-derived galectin-9 may be a novel biomarker for predicting and monitoring liver ACR.

Liver biopsy remains the gold standard for ACR diagnosis, but its expense, inconvenience, and invasiveness ultimately highlight the need for noninvasive and reliable diagnostic biomarkers. Exosome biomarkers have been widely studied in cancers. Cancer cells and immune cells in the cancer microenvironment release exosomes into the periphery to deliver specific signals. Thus, exosomes from cancers carry molecular signatures of pathophysiological status and different stages of progression (Mousavi et al., 2019; Naderi-Meshkin et al., 2019). Lim et al. (2018) used urinary exosomes to evaluate kidney transplant rejection, suggesting that exosome-derived tetraspanin-1 and hemopexin are significantly higher in patients with ACR. Dieudé et al. (2015) showed that apoptotic exosome-like vesicles and their contents are associated with graft rejection after transplantation. These apoptotic exosome-like vesicles induce anti-perlecan antibodies and allograft inflammation in a mouse model of MHC-incompatible aortic graft transplantation, and the active $20 \mathrm{~S}$ proteasome core complexes in apoptotic exosome-like vesicles play a key role in this process (Dieudé et al., 2015). To our knowledge, there have been no previous studies using exosomes to evaluate liver rejection. In the present study, we found high galectin-9 expression in circulating exosome in patients with ACR, which indicates that it may serve as a biomarker for rejection and survival.

Galectin-9 is abundantly expressed in the liver and is involved in determining liver rejection or tolerance (Tang et al., 2013; Huang et al., 2018). Galectin-9 promotes the production of pro-inflammatory cytokines, including IFN- $\gamma$ and tumor necrosis factor $\alpha$ (TNF- $\alpha$ ) in helper T cells (Su et al., 2011), and Tim-3 is closely related to $\mathrm{T}$ cells in allograft transplantation (Gupta et al., 2012). Furthermore, the N-terminal region of galectin-9 is effective in dendritic cell (DC) activation, by inducing TNF- $\alpha$ and IL-6 (ChaganYasutan et al., 2010). T cells and DCs are known to be key regulators of ACR, and therefore, galectin-9 may be important in mediating rejection. In fact, previous studies have shown that the galectin-9/ Tim-3 pathway negatively regulates $\mathrm{T}$ cell responses (Dardalhon et al., 2010; Sakuishi et al., 2011), plays an important role in ACR, and is associated with rejection severity (Naka et al., 2009). Our results are in line with these findings, since high levels of galectin-9 were found in exosomes from patients with ACR. However, age and Child-Pugh score were also different between the rejection and non-rejection groups (Table 2). Therefore, understanding the detailed function of galectin-9 in liver allograft rejection requires further investigation.

The status of the original disease and its consequent systemic pathophysiological state are associated with transplant patient outcome (Schlegel et al., 2017). The Cox regression analysis performed in the present study included some important indices, such as age, gender, Child-Pugh score, serum creatinine concentration, and galectin-9 expression. The results of this analysis showed that galectin-9 expression in the diseased liver was an independent factor predicting the long-term survival of patients. ABO incompatibility is regarded as a risk factor for antibodymediated rejection and poor outcome (Lee et al., 2017). In the present study, ABO incompatibility was not an independent risk factor for rejection or prognosis. This may be due to the use of rituximab by these patients (Shen et al., 2014).

There are several limitations in this study. Firstly, although all the patients included in the study had benign end-stage liver disease, the subjects consisted of those with hepatitis B infection (HBV)/ethanol alcohol $(\mathrm{EtOH})$-related cirrhosis and metabolic disorders. Therefore, different individual patients may have different pathological backgrounds that may affect exosome contents and galectin-9 expression. Secondly, ACR is the result of immune reactions between the recipient and the donor liver. However, galectin-9 expression was only examined in the recipient and the influence of the donor's galectin-9 expression level was not investigated. The third limitation is the small sample size and in particular, the limited number used for exosome isolation and identification. 


\section{Conclusions}

In the present study, we employed exosome and galectin-9 for acute rejection evaluation. Our results showed that the level of intrinsic galectin-9 expression in the resected livers of transplant recipients may be a marker of recipient immune status and associated with post-transplant rejection. Exosome-derived galectin-9 levels may be a novel predictor of rejection and prognosis after liver transplantation.

\section{Contributors}

Ai-bin ZHANG: study design, data analysis, and manuscript writing. Yi-fan PENG: data collection and manuscript writing. Jun-jun JIA: study design and data analysis. Yu NIE and Shi-yu ZHANG: data collection and data analysis. Hai-yang XIE and Lin ZHOU: manuscript revision. Shu-sen ZHENG: conception direction and study design.

\section{Compliance with ethics guidelines}

Ai-bin ZHANG, Yi-fan PENG, Jun-jun JIA, Yu NIE, Shi-yu ZHANG, Hai-yang XIE, Lin ZHOU, and Shu-sen ZHENG declare that they have no conflict of interest.

All procedures followed were in accordance with the ethical standards of the responsible committee on human experimentation (institutional and national) and with the Helsinki Declaration of 1975, as revised in 2008 (5). Informed consent was obtained from all patients for being included in the study.

\section{References}

Ashokkumar C, Soltys K, Mazariegos G, et al., 2017. Predicting cellular rejection with a cell-based assay: preclinical evaluation in children. Transplantation, 101(1):131-140. https://doi.org/10.1097/tp.0000000000001076

Boix F, Millan O, san Segundo D, et al., 2016. High expression of CD38, CD69, CD95 and CD154 biomarkers in cultured peripheral $\mathrm{T}$ lymphocytes correlates with an increased risk of acute rejection in liver allograft recipients. Immunobiology, 221(5):595-603. https://doi.org/10.1016/j.imbio.2016.01.008

Chagan-Yasutan H, Shiratori B, Siddiqi UR, et al., 2010. The increase of plasma galectin-9 in a patient with insulin allergy: a case report. Clin Mol Allergy, 8:12. https://doi.org/10.1186/1476-7961-8-12

Chen DJ, Peng WH, Jiang H, et al., 2017. Noninvasive detection of acute renal allograft rejection by measurement of soluble Tim-3 in urine. Mol Med Rep, 16(1):915-921. https://doi.org/10.3892/mmr.2017.6670

Dardalhon V, Anderson AC, Karman J, et al., 2010. Tim-3/ galectin-9 pathway: regulation of Th1 immunity through promotion of $\mathrm{CD} 11 \mathrm{~b}^{+} \mathrm{Ly}-6 \mathrm{G}^{+}$myeloid cells. J Immunol, 185(3):1383-1392.

https://doi.org/10.4049/jimmunol.0903275
Demetris AJ, Bellamy C, Hübscher SG, et al., 2016. 2016 comprehensive update of the Banff Working Group on Liver Allograft Pathology: introduction of antibodymediated rejection. Am J Transplant, 16(10):2816-2835. https://doi.org/10.1111/ajt.13909

Dieudé M, Bell C, Turgeon J, et al., 2015. The 20S proteasome core, active within apoptotic exosome-like vesicles, induces autoantibody production and accelerates rejection. Sci Transl Med, 7(318):318ra200. https://doi.org/10.1126/scitranslmed.aac9816

Gupta S, Thornley TB, Gao WD, et al., 2012. Allograft rejection is restrained by short-lived TIM- $3^{+} \mathrm{PD}-1^{+}$Foxp $3^{+}$ Tregs. J Clin Invest, 122(7):2395-2404. https://doi.org/10.1172/jci45138

Huang HT, Lu YF, Zhou T, et al., 2018. Innate immune cells in immune tolerance after liver transplantation. Front Immunol, 9:2401. https://doi.org/10.3389/fimmu.2018.02401

Kerr N, García-Contreras M, Abbassi S, et al., 2018. Inflammasome proteins in serum and serum-derived extracellular vesicles as biomarkers of stroke. Front $\mathrm{Mol}$ Neurosci, 11:309. https://doi.org/10.3389/fnmol.2018.00309

Kim N, Yoon YI, Yoo HJ, et al., 2016. Combined detection of serum IL-10, IL-17, and CXCL10 predicts acute rejection following adult liver transplantation. Mol Cells, 39(8): 639-644. https://doi.org/10.14348/molcells.2016.0130

Kurose Y, Wada J, Kanzaki M, et al., 2013. Serum galectin-9 levels are elevated in the patients with type 2 diabetes and chronic kidney disease. BMC Nephrol, 14:23. https://doi.org/10.1186/1471-2369-14-23

Lazar E, Benedek T, Korodi S, et al., 2018. Stem cell-derived exosomes - an emerging tool for myocardial regeneration. World J Stem Cells, 10(8):106-115. https://doi.org/10.4252/wjsc.v10.i8.106

Lee EC, Kim SH, Park SJ, 2017. Outcomes after liver transplantation in accordance with $\mathrm{ABO}$ compatibility: a systematic review and meta-analysis. World J Gastroenterol, 23(35):6516-6533. https://doi.org/10.3748/wjg.v23.i35.6516

Li YM, Shi YY, Li Y, et al., 2018. Soluble Tim-3 and Gal-9 are associated with renal allograft dysfunction in kidney transplant recipients: a cross-sectional study. Int Immunopharmacol, 55:330-335. https://doi.org/10.1016/j.intimp.2018.01.008

Lim JH, Lee CH, Kim KY, et al., 2018. Novel urinary exosomal biomarkers of acute $\mathrm{T}$ cell-mediated rejection in kidney transplant recipients: a cross-sectional study. PLoS ONE, 13(9):e0204204. https://doi.org/10.1371/journal.pone.0204204

Meszaros M, Ursic-Bedoya J, Faure S, et al., 2019. Immunosuppression minimization trials in liver transplantation: can we predict humoral response to assess eligibility? Hepatology, 69(5):2302-2303. https://doi.org/10.1002/hep.30345 
Mousavi S, Moallem R, Hassanian SM, et al., 2019. Tumorderived exosomes: potential biomarkers and therapeutic target in the treatment of colorectal cancer. J Cell Physiol, 234(8):12422-12432. https://doi.org/10.1002/jcp.28080

Naderi-Meshkin H, Lai X, Amirkhah R, et al., 2019. Exosomal lncRNAs and cancer: connecting the missing links. Bioinformatics, 35(2):352-360. https://doi.org/10.1093/bioinformatics/bty527

Naka EL, Ponciano VC, Cenedeze MA, et al., 2009. Detection of the Tim-3 ligand, galectin-9, inside the allograft during a rejection episode. Int Immunopharmacol, 9(6):658-662. https://doi.org/10.1016/j.intimp.2008.11.013

Pike R, Thomas N, Workman S, et al., 2016. PD1-expressing T cell subsets modify the rejection risk in renal transplant patients. Front Immunol, 7:126. https://doi.org/10.3389/fimmu.2016.00126

Qiao XW, Jiang K, Nie J, et al., 2014. Increased expression of Tim- 3 and its ligand galectin-9 in rat allografts during acute rejection episodes. Biochem Biophys Res Commun, 445(3):542-548. https://doi.org/10.1016/j.bbrc.2014.01.167

Raschzok N, Reutzel-Selke A, Schmuck RB, et al., 2015. CD44 and CXCL9 serum protein levels predict the risk of clinically significant allograft rejection after liver transplantation. Liver Transpl, 21(9):1195-1207. https://doi.org/10.1002/1t.24164

Saitoh H, Ashino Y, Chagan-Yasutan H, et al., 2012. Rapid decrease of plasma galectin-9 levels in patients with acute HIV infection after therapy. Tohoku J Exp Med, 228(2): 157-161. https://doi.org/10.1620/tjem.228.157

Sakuishi K, Jayaraman P, Behar SM, et al., 2011. Emerging Tim-3 functions in antimicrobial and tumor immunity. Trends Immunol, 32(8):345-349. https://doi.org/10.1016/j.it.2011.05.003

Schlegel A, Linecker M, Kron P, et al., 2017. Risk assessment in high- and low-meld liver transplantation. Am J Transplant, 17(4):1050-1063. https://doi.org/10.1111/ajt.14065

Shen T, Lin BY, Jia JJ, et al., 2014. A modified protocol with rituximab and intravenous immunoglobulin in emergent ABO-incompatible liver transplantation for acute liver failure. Hepatobiliary Pancreat Dis Int, 13(4):395-401. https://doi.org/10.1016/S1499-3872(14)60268-X

Sood S, Haifer C, Yu LJ, et al., 2017. A novel immune function biomarker identifies patients at risk of clinical events early following liver transplantation. Liver Transpl, 23(4):487-497. https://doi.org/10.1002/lt.24730

Su EW, Bi SG, Kane LP, 2011. Galectin-9 regulates T helper cell function independently of Tim-3. Glycobiology, 21(10): 1258-1265.

https://doi.org/10.1093/glycob/cwq214

Tang ZH, Liang SW, Potter J, et al., 2013. Tim-3/galectin-9 regulate the homeostasis of hepatic NKT cells in a murine model of nonalcoholic fatty liver disease. $J$ Immunol, 190(4):1788-1796. https://doi.org/10.4049/jimmunol.1202814

Vallabhajosyula P, Korutla L, Habertheuer A, et al., 2017. Tissue-specific exosome biomarkers for noninvasively monitoring immunologic rejection of transplanted tissue. J Clin Invest, 127(4):1375-1391. https://doi.org/10.1172/jci87993

van den Hoogen LL, van Roon JAG, Mertens JS, et al., 2018. Galectin-9 is an easy to measure biomarker for the interferon signature in systemic lupus erythematosus and antiphospholipid syndrome. Ann Rheum Dis, 77(12): 1810-1814. https://doi.org/10.1136/annrheumdis-2018-213497

Vitalone MJ, Wei L, Fujiki M, et al., 2016. Liver microRNA profile of induced allograft tolerance. Transplantation, 100(4):781-790. https://doi.org/10.1097/TP.0000000000001105

Wang Y, Zhang M, Liu ZW, et al., 2014. The ratio of circulating regulatory $\mathrm{T}$ cells (Tregs)/Th17 cells is associated with acute allograft rejection in liver transplantation. PLOS ONE, 9(11):e112135. https://doi.org/10.1371/journal.pone.0112135

Zhou YX, Yang XJ, Zhang H, et al., 2015. The roles of T helper type 17/regulatory $T$ cells in acute rejection after liver transplantation in rats. Transplantation, 99(6):11261131. https://doi.org/10.1097/TP.0000000000000666

\section{中文概要}

题 目: 外泌体来源的半乳糖凝集素-9 预测肝移植术后排 斥发生及预后

目 的: 外泌体及其内容物是各种肝脏疾病的潜在生物标 志物。本研究探索外泌体及其内容物在肝移植排 斥反应及预后中的作用。

创新点: 本研究发现外泌体及内含物半乳糖凝集素-9 (galectin-9) 在肝移植术后排斥及预后预测中发 挥重要作用。

方 法: 分别从急性排斥和肝功能稳定患者提取外泌体, 进行分离、鉴定并检测其内含蛋白。候选蛋白通 过在 73 个急性排斥病人和 63 个肝功能稳定病人 切除肝的组织芯片中进行验证。最后将蛋白表达 量和临床参数纳入 Kaplan-Meier 生存率和 Cox 回 归分析。

结 论: 外泌体来源的 galectin-9 可作为预测肝移植术后 排斥发生及预后的生物学指标。

关键词: 肝移植; 急性排斥反应; 外泌体; 半乳糖凝集素-9 (Galectin-9) 